\title{
Isolation and characterization of erlotinib-resistant human non-small cell lung cancer A549 cells
}

\author{
RYUJI IKEDA ${ }^{1}$, LEE C. VERMEULEN ${ }^{2}$, ELIM LAU $^{3}$, ZHISHENG JIANG $^{3}$, \\ SHANNON M. KAVANAUGH ${ }^{3}$, KATSUSHI YAMADA ${ }^{1}$ and JILL M. KOLESAR ${ }^{3}$ \\ ${ }^{1}$ Department of Clinical Pharmacy and Pharmacology, Graduate School of Medical and Dental Sciences, \\ Kagoshima University, Kagoshima, Japan; ${ }^{2}$ Division of Pharmacy Practice, School of Pharmacy, \\ University of Wisconsin-Madison, Madison, and Center for Drug Policy, University of Wisconsin Hospital and Clinics; \\ ${ }^{3}$ School of Pharmacy, University of Wisconsin-Madison, and \\ University of Wisconsin Paul P. Carbone Comprehensive Cancer Center, Madison, WI, USA
}

Received July 27, 2010; Accepted September 16, 2010

DOI: $10.3892 / \mathrm{ol} .2010 .198$

\begin{abstract}
Erlotinib, an epidermal growth factor receptor (EGFR) tyrosine kinase inhibitor, is an effective therapy for non-small cell lung cancer (NSCLC). However, resistance to erlotinib reduces its efficacy. To investigate the basis of erlotinib resistance, we isolated erlotinib-resistant human NSCLC A549 cells, termed A549/ER cells. The A549/ ER cells were found to be resistant to erlotinib, as well as paclitaxel and gemcitabine. We then performed a PCR array to investigate the resistance to erlotinib in A549/ER cells. EGFR expression in A549/ER cells was decreased compared to A549 cells. The expression of fibroblast growth factor 2 (FGF2) and p21 in A549/ER was increased when compared to A549 cells. Our results suggest that the down-regulation of EGFR and up-regulation of FGF2 is related to resistance to erlotinib in A549/ER cells.
\end{abstract}

\section{Introduction}

Non-small cell lung cancer (NSCLC) is a significant cause of cancer-related mortality in the US and worldwide (1). Epidermal growth factor receptor (EGFR) is overexpressed in various epithelial types of cancer, including NSCLC, and its inhibitors have been investigated as first-line or subsequent therapy options for patients with advanced metastatic NSCLC (2). Two classes of EGFR inhibitor, monoclonal antibodies, including cetuximab, and small-molecule tyrosine kinase inhibitors (TKIs), including erlotinib and gefitinib, have been

Correspondence to: Professor Jill M. Kolesar, School of Pharmacy, University of Wisconsin, and University of Wisconsin Paul P. Carbone Comprehensive Cancer Center, 600 Highland Avenue, Room K4/554, Madison, WI 53705, USA

E-mail: jmkolesar@pharmacy.wisc.edu

Key words: erlotinib, epidermal growth factor receptor, fibroblast growth factor 2, p21 studied in phase III trials and are used clinically to treat NSCLC (3-8). Although erlotinib is an effective therapy for NSCLC, resistance to erlotinib reduces its efficacy. Erlotinib resistance is associated with changes in EGFR itself or in the expression of other genes (9). In particular, the T790M mutation has been reported in $50 \%$ of EGFR TKI-resistant tumors $(10,11)$. To investigate the basis of erlotinib resistance, erlotinib-resistant human NSCLC A549 cells, termed A549/ER cells, were isolated. A PCR array was performed to identify erlotinib resistance in A549/ER cells.

\section{Materials and methods}

Reagents. Paclitaxel, ethyl methanesulfonate and 3-[4, 5-dimethylthiazol-2-yl]-2,5-diphenyltetrazolium bromide (MTT) were obtained from Sigma Chemical Co. (St. Louis, MO, USA). The gemcitabine hydrochloride used was from LKT Laboratories, Inc. (MN, USA). Erlotinib was from Chemie Tek (Indianapolis, IN, USA). Fetal bovine serum (FBS), Dulbecco's modified Eagle's medium (DMEM), penicillin-streptomycin solution $(10,000 \mathrm{U} / \mathrm{ml}$ penicillin and $10,000 \mu \mathrm{g} / \mathrm{ml}$ streptomycin) were from Hyclone (UT, USA). RT-2 Profiler PCR Array Human Cancer Drug Resistance and Metabolism (PAHS-004A-2) was from SA Biosciences (Frederick, MD, USA).

Cell culture. The A549 cell line, derived from NSCLC, was maintained in DMEM containing 10\% FBS, $100 \mathrm{U} / \mathrm{ml}$ penicillin and $100 \mu \mathrm{g} / \mathrm{ml}$ streptomycin at $37^{\circ} \mathrm{C}$ in a $5 \% \mathrm{CO}_{2}$ humidified atmosphere.

Cell proliferation by 3-[4, 5-dimethylthiazol-2-yl]-2, 5-diphenyltetrazolium bromide assay. Cell proliferation in vitro was measured using a MTT colorimetric assay in 96-well plates. The cells $\left(5 \times 10^{3}\right)$ were inoculated into each well. Following overnight incubation $\left(37^{\circ} \mathrm{C}\right.$ in $\left.5 \% \mathrm{CO}_{2}\right)$, anti-cancer agents were added to the cultured and incubated for 3 days. A total of $50 \mu \mathrm{l}$ of MTT $(1 \mathrm{mg} / \mathrm{ml})$ was added to each well and the plates were incubated for an additional $4 \mathrm{~h}$. Following aspiration of the culture medium, the resulting formazan was dissolved with 


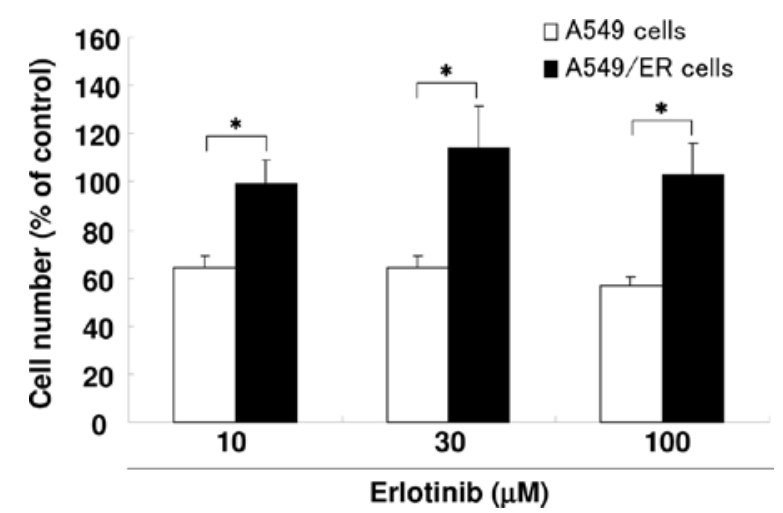

Figure 1. Sensitivity of erlotinib in A549 and A549/ER cells. A549 and A549/ER cells were treated with various concentrations of erlotinib for $72 \mathrm{~h}$ and cell viability was determined using MTT assay in each cell line. The columns and bars are the mean $\pm \mathrm{SD}$. ${ }^{*} \mathrm{P}<0.05$.

$100 \mu \mathrm{l}$ of dimethylsulfoxide. The plates were read at $570 \mathrm{~nm}$ using a microplate reader.

Chronic erlotinib exposure. Erlotinib-resistant A549/ER cells were isolated by the A549 cells with increasing concentrations of erlotinib following ethyl methanesulfonate-induced mutagenesis, and then incubated in a selection medium with erlotinib (1-100 $\mu \mathrm{M})$.

Reverse transcription-polymerase chain reaction (RT-PCR) method. Total cellular RNA was extracted by the RNeasy Mini kit (Qiagen Sciences, MD, USA). RNA quality and concentration were confirmed in NanoDrop ND-100 Spectrophotometer (Thermo Scientific, Wilmington, DE, USA). For RT-PCR, $1 \mu \mathrm{g}$ of total RNA was used for cDNA synthesis using an iScript cDNA synthesis kit (Bio-Rad, CA, USA), according to the manufacturer's instructions. The conditions for the RT-PCR were: $5 \mathrm{~min}$ at $95^{\circ} \mathrm{C}$ and then 28 cycles of amplification in PCR master mix (Promega, WI, USA) at $95^{\circ} \mathrm{C}$ for $30 \mathrm{sec}$, annealing at $52^{\circ} \mathrm{C}$ for $30 \mathrm{sec}$ and extension at $72^{\circ} \mathrm{C}$ for $1 \mathrm{~min}$. The primers used for this analysis were: p21, forward 5'-ctcttcggccagtggacagc-3' and reverse 5'-agagtctccaggtccacctgg-3'; fibroblast growth factor 2 (FGF2), forward 5'-ccttgccegaggatggcggca-3' and reverse 5'-ttgaccggtaagtattgtagt-3'; EGFR, forward 5'-gccacaggccaggt ctgccat-3' and reverse 5-ccggcgtctgcgtacttccag-3; and GAPDH, forward 5'-gtcttcaccaccatggagaagg-3' and reverse 5'-ggcaggt caggtccaccactga- 3 '.

Genomic DNA extraction and single nucleotide polymorphism genotyping. Genomic DNA from A549 and A549/ER cells were extracted with a QIAamp DNA Mini kit (Qiagen Sciences). The conditions for the RT-PCR were: $5 \mathrm{~min}$ at $95^{\circ} \mathrm{C}$ and then 55 cycles of amplification in PCR master mix (Promega) at $95^{\circ} \mathrm{C}$ for $30 \mathrm{sec}$, annealing at $52^{\circ} \mathrm{C}$ for $30 \mathrm{sec}$ and extension at $72^{\circ} \mathrm{C}$ for $1 \mathrm{~min}$. The mutations were genotyped using PSQ96MA (Qiagen, Germantown, MD, USA).

The primers used for this analysis were: T790M, forward 5'-tgggcatctgcctcacct-3', reverse 5'-ctttgtgttcceggacat-3' and sequence primer 5'-cctcacctccaccgt-3'; L861Q, forward 5'-agc caggaacgtactggtgaa-3', reverse 5'-gcctccttctgcatggtattc-3' and sequence primer 5'-tcacagattttgggc-3'.
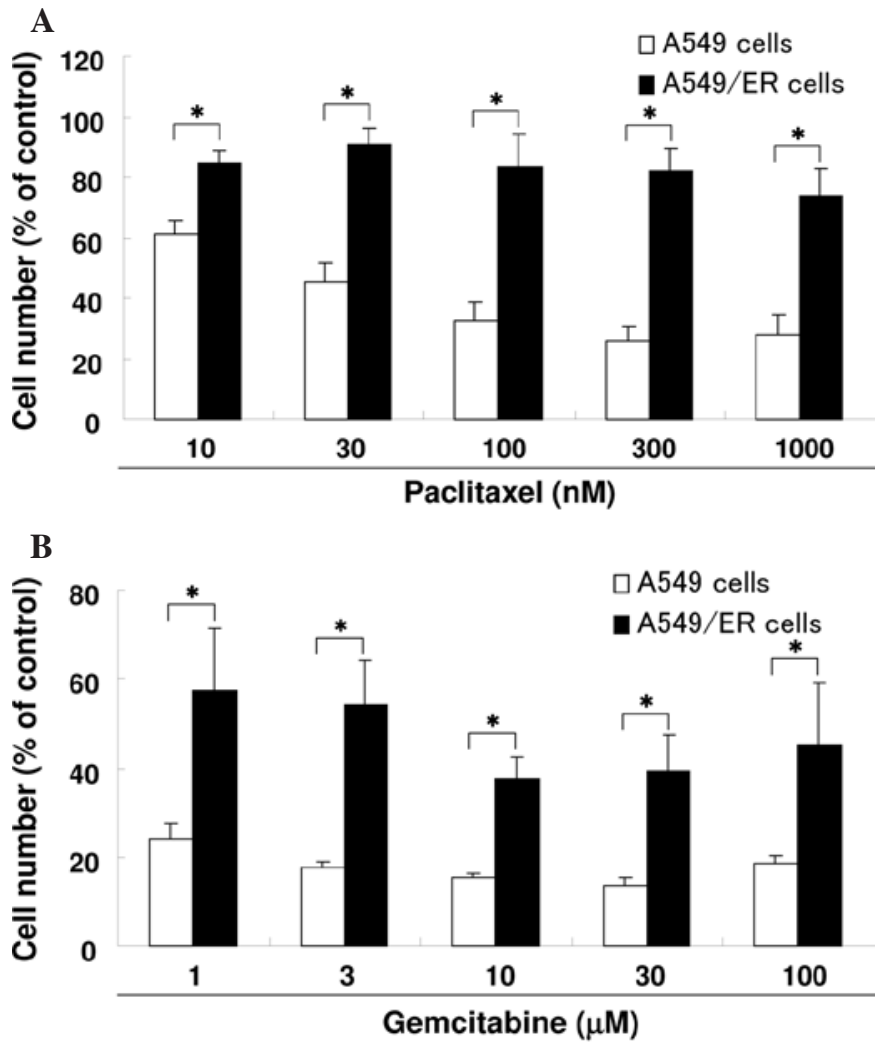

Figure 2. Sensitivity of A549 and A549/ER cells to paclitaxel and gemcitabine. A549 and A549/ER cells were treated with various concentrations of (A) paclitaxel and (B) gemcitabine for $72 \mathrm{~h}$ and cell viability was determined using MTT assay in each cell line. The columns and bars are the mean \pm SD. ${ }^{*} \mathrm{P}<0.05$.

PCR array. Total cellular RNA was extracted by the RNeasy Mini kit (Qiagen Sciences). RNA quality and concentration were confirmed in the NanoDrop ND-100 Spectrophotometer (Thermo Scientific). For RT-PCR, $1 \mu \mathrm{g}$ of total RNA was used for cDNA synthesis using the iScript cDNA synthesis kit (Bio-Rad), according to the manufacturer's instructions. After cDNAs were mixed with SYBR-Green Supermix, the mixtures were added to the plates of RT-2 Profiler PCR Array Human Cancer Drug Resistance and Metabolism. The conditions for real-time PCR were: $10 \mathrm{~min}$ at $95^{\circ} \mathrm{C}$ and then 40 cycles at $95^{\circ} \mathrm{C}$ for $15 \mathrm{sec}$ and at $60^{\circ} \mathrm{C}$ for $1 \mathrm{~min}$. The data from PCR array were normalized according to the manufacturer's guidelines using software from SA Bioscience.

Statistical analysis. Data are presented as the mean \pm SD. Statistical analysis was performed using StatView 5.0. (SAS Institute Inc., Cary, NC, USA). Differences were considered significant at $\mathrm{P}<0.05$.

\section{Results}

Establishment of erlotinib-resistant non-small cell lung cancer A549 cells. To isolate erlotinib-resistant A549/ER cells, A549 cells were cultured in stepwise selection containing an increasing erlotinib concentration, from 10 to $100 \mu \mathrm{M}$. The sensitivity to erlotinib was examined in each cell line. Results showed that the A549/ER cells were more resistant than the parental A549 cells (Fig. 1). 
A

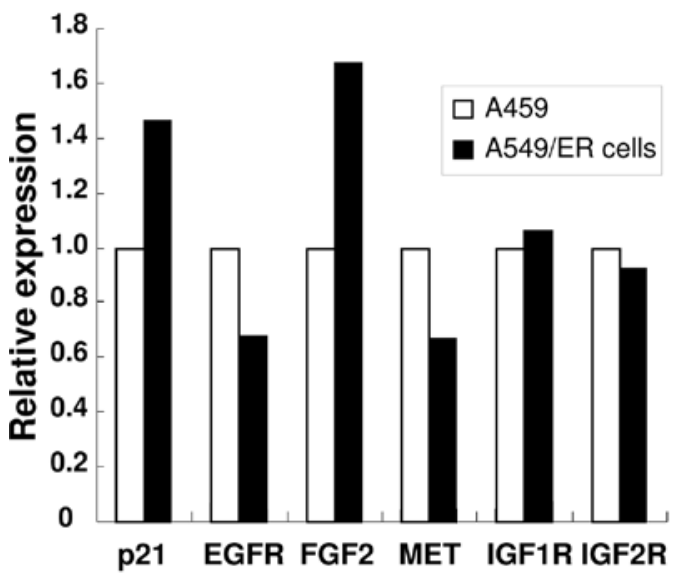

B

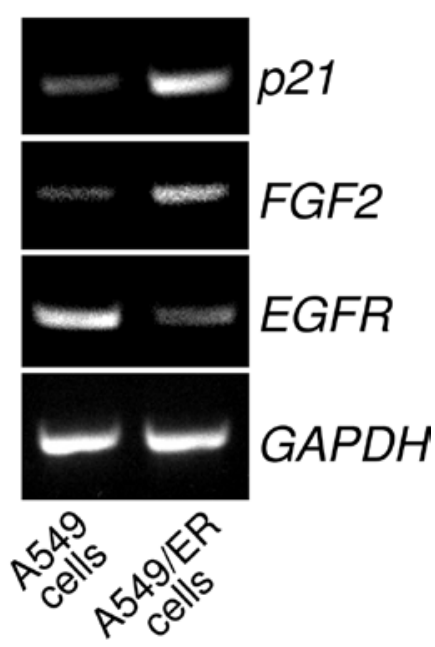

Figure 3. Expression of EGFR, MET, p21 and FGF2 in A549 and A549/ ER cells. (A) Expression of $p 21, E G F R, M E T, F G F 2, I G F 1 R$ and $I G F 2 R$ in A549 and A549/ER cells was detected by PCR array. Following preparation of the total RNA, $1 \mu \mathrm{g}$ was used for cDNA synthesis. The expression of genes was determined by PCR array, as described in Materials and methods Data are normalized to the expression of each gene in parental A549 cells (B) Expression of EGFR, FGF2 and $p 21$ in A549 and A549/ER cells. The expression of EGFR, FGF2, p21 and GAPDH in A549 and A549/ER cells was measured using RT-PCR. GAPDH expression was detected as a control.

EGFR mutation in A549/ER cells. EGFR mutations, including the threonine-to-methionine substitution at position 790 (T790M, in exon 20) and the leucine-to-glutamine (L861Q, in exon 21) have been reported to be resistance mutations to erlotinib (12). To examine T790M and L861Q mutations in A549/ER cells, pyrosequencing was used. The EGFR T790M and L861Q mutations were not present in the A549/ER cells (data not shown).

Cross resistance to erlotinib. The drug sensitivity of each cell line was tested using a MTT assay. Fig. 2 shows the sensitivity of the parental and resistant cell lines to various anti-cancer agents. Notably, A549/ER cells were resistant to paclitaxel and gemcitabine as compared to A549 cells (Fig. 2A and B).

PCR array analysis and RT-PCR analysis of A549 and A549/ $E R$ cells. The temporal pattern of gene expression in A549 and A549/ER cells was compared by a PCR array that covered 84 genes. A number of genes including EGFR and MET were down-regulated in A549/ER compared to A549 cells. A total of five genes were up-regulated more than 1.4-fold in A549/ER cells. The genes, including the ones for $F G F 2$ and $p 21 / C D K N 1 A$, were increased in A549/ER compared to A549 cells (Fig. 3A). The expression of insulin-like growth factor-1 receptor $(I G F 1 R)$ and IGF2R was not altered between A549 and A549/ER cells. To validate the PCR array using independent methods, RT-PCR analysis was performed using specific primers for each gene. Consistent with the array data, FGF2 and $p 21$ expression was increased in A549 cells, while $E G F R$ expression was decreased (Fig. 3B).

\section{Discussion}

Lung cancer is the most common cause of cancer-related mortality in developed nations. NSCLC normally presents as incurable locally advanced or metastatic disease. Despite significant research efforts, survival prospects remain poor and $14 \%$ of all patients with lung cancer are expected to live 5 years after diagnosis (1). EGFR-specific TKIs, including gefitinib and erlotinib, have been developed as therapeutic agents for NSCLC treatment $(6,7)$. Although the benefits of erlotinib were statistically significant, resistance to erloninib reduces its efficacy. To investigate the basis of resistance to erlotinib, we isolated erlotinib-resistant human NSCLC A549 cells, termed A549/ER cells.

One mechanism of acquired resistance occurs when EGFR is mutated. Mutations at T790M and L861Q are normally associated with erlotinib resistance (12). Therefore, we performed a sequence of genome in A549 cells and A549/ER cells. Results showed that neither A549 nor A549/ER cells carried a mutation of T790M and L861Q; thus, the acquired resistance identified in this study was not caused by EGFR mutation.

To investigate the basis of resistance to erlotinib, we performed a PCR array in A549 and A549/ER cells. The expression of EGER and MET in A549/ER cells was decreased when compared to the sensitive A549 cells. Additionally, similar levels of IGFIR and IGF2R were expressed in the two cell lines. EGFR signaling is linked to multiple intracellular pathways that inhibit apoptosis and promote survival and proliferation. Upon ligand-induced activation, EGFR and MET generate phosphotyrosine sites for the recruitment of Ras and phosphatidylinositol-3 kinase (PI3K), resulting in the classic mitogen-activated protein kinase and Akt pathway (13). These results suggest that A549/ER cells are independent of EGFR signaling for their growth and survival. Notably, the expression of FGF2 in A549/ER cells was increased compared to that in A549 cells. Through the activation of FGFR1 $\beta$ signaling, FGF2 promotes the survival and proliferation of tumors. Stimulation of FGFR1 $\beta$ results in PI3K/ Akt activation and causes resistance to anti-cancer agents $(14,15)$. Neutralizing FGFR1-specific antibody abrogates the physiologic and chemoprotective effects of FGF2/FGFR1 $\beta$ signaling (15). The A549/ER cells may have alternative means of maintaining PI3K/Akt signaling, including FGF2/ FGFR overexpression, that activates PI3K/Akt signaling in an EGFR- or MET-independent manner. Strategies, including the neutralizing FGFR1 or FGF2-specific antibody, or inhibitors of FGFR1, may be effective in restoring sensitivity to erlotinib-resisitant cells. 
On the other hand, the overexpression of p21 inhibits proliferation in mammalian cells and has been found to inhibit all cyclin-CDK complexes, suggesting that it is a cyclin-CDK inhibitor (16). p21 has demonstrated that the inhibitory control on cyclin-CDK complexes is mediated through its N-terminal domain and is distinct from its ability to bind PCNA (16). p21 plays an essential role in growth arrest after DNA damage, while its overexpression leads to cell cycle arrest, which prevents DNA damage (17). Erlotinib-mediated signaling is involved in the up-regulation of p21 (18). We demonstrated the overexpression of $p 21$ in erlotinib-resistant cells (A549/ ER cells) when compared to A549 cells (Fig. 3). Additionally, Ferrandiz et al have reported that p21-deficient colon cancer cells were more sensitive towards imatinib and gefitinib than parental cells (19). These results suggest that the overexpression of p21 may prevent erlotinib signaling in A549/ER cells.

In conclusion, a better understanding of the characterization and mechanism of resistance to erlotinib in A549/ER cells may be useful in the identification of agents that reverse clinical erlotinib resistance in NSCLC.

\section{Acknowledgements}

The authors would like to thank the University of Wisconsin Carbone Comprehensive Cancer Center (UWCCC) for use of its facilities to complete this study. The study is supported in part by NIH/NCI P30 CA014520 - UW Comprehensive Cancer Center Support.

\section{References}

1. Azzoli CG, Baker S Jr, Temin S, et al: American Society of Clinical Oncology Clinical Practice Guideline update on chemotherapy for stage IV non-small-cell lung cancer. J Clin Oncol 27: 6251-6266, 2009.

2. Hirsch FR, Varella-Garcia M and Cappuzzo F: Predictive value of EGFR and HER2 overexpression in advanced non-small-cell lung cancer. Oncogene 28: 32-37, 2009.

3. Ciardiello $\mathrm{F}$ and Tortora G: EGFR antagonists in cancer treatment. N Engl J Med 358: 1160-1174, 2008.

4. Hynes NE and Lane HA: ERBB receptors and cancer: the complexity of targeted inhibitors. Nat Rev Cancer 5: 341-354, 2005 .

5. Mendelsohn J and Baselga J: Status of epidermal growth factor receptor antagonists in the biology and treatment of cancer. J Clin Oncol 21: 2787-2799, 2003.

6. Kelly K, Chansky K, Gaspar LE, Albain KS, Jett J, Ung YC, Lau DH, Crowley JJ and Gandara DR: Phase III trial of maintenance gefitinib or placebo after concurrent chemoradiotherapy and docetaxel consolidation in inoperable stage III non-smallcell lung cancer: SWOG S0023. J Clin Oncol 26: 2450-2456, 2008.
7. Gatzemeier U, Pluzanska A, Szczesna A, et al: Phase III study of erlotinib in combination with cisplatin and gemcitabine in advanced non-small-cell lung cancer: the Tarceva Lung Cancer Investigation Trial. J Clin Oncol 25: 1545-1552, 2007.

8. Pirker R, Pereira JR, Szczesna A, von Pawel J, Krzakowski M, Ramlau R, Vynnychenko I, Park K, Yu CT, Ganul V, Roh JK, Bajetta E, O'Byrne K, de Marinis F, Eberhardt W, Goddemeier T, Emig M, Gatzemeier U; FLEX Study Team: Cetuximab plus chemotherapy in patients with advanced non-small-cell lung cancer (FLEX): an open-label randomised phase III trial. Lancet 373: 1525-1531, 2009.

9. Gazdar AF: Epidermal growth factor receptor inhibition in lung cancer: the evolving role of individualized therapy. Cancer Metastasis Rev 29: 37-48, 2010.

10. Kosaka T, Yatabe Y, Endoh H, Yoshida K, Hida T, Tsuboi M, Tada H, Kuwano $\mathrm{H}$ and Mitsudomi T: Analysis of epidermal growth factor receptor gene mutation in patients with non-small cell lung cancer and acquired resistance to gefitinib. Clin Cancer Res 12: 5764-5769, 2006.

11. Balak MN, Gong Y, Riely GJ, Somwar R, Li AR, Zakowski MF, Chiang A, Yang G, Ouerfelli O, Kris MG, Ladanyi M, Miller VA and Pao W: Novel D761Y and common secondary T790M mutations in epidermal growth factor receptor-mutant lung adenocarcinomas with acquired resistance to kinase inhibitors. Clin Cancer Res 12: 6494-6501, 2006.

12. Kancha RK, von Bubnoff N, Peschel C and Duyster J: Functional analysis of epidermal growth factor receptor (EGFR) mutations and potential implications for EGFR targeted therapy. Clin Cancer Res 15: 460-467, 2009.

13. Pawson T: Specificity in signal transduction: from phosphotyrosine-SH2 domain interactions to complex cellular systems. Cell 116: 191-203, 2004.

14. Song S, Wientjes MG, Gan Y and Au JL: Fibroblast growth factors: an epigenetic mechanism of broad spectrum resistance to anticancer drugs. Proc Natl Acad Sci USA 97: 8658-8663, 2000.

15. Karajannis MA, Vincent L, Direnzo R, Shmelkov SV, Zhang F, Feldman EJ, Bohlen P, Zhu Z, Sun H, Kussie P and Rafii S: Activation of FGFR1beta signaling pathway promotes survival, migration and resistance to chemotherapy in acute myeloid leukemia cells. Leukemia 20: 979-986, 2006.

16. Abukhdeir AM and Park BH: P21 and p27: roles in carcinogenesis and drug resistance. Expert Rev Mol Med 10: e19, 2008.

17. Gartel AL and Tyner AL: The role of the cyclin-dependent kinase inhibitor p21 in apoptosis. Mol Cancer Ther 1: 639-649, 2002.

18. Sutter AP, Höpfner M, Huether A, Maaser K and Scherübl H: Targeting the epidermal growth factor receptor by erlotinib (Tarceva) for the treatment of esophageal cancer. Int J Cancer 118: 1814-1822, 2006.

19. Ferrandiz N, Martin-Perez J, Blanco R, Donertas D, Weber A, Eilers M, Dotto P, Delgado MD and Leon J: HCT116 cells deficient in p21(Waf1) are hypersensitive to tyrosine kinase inhibitors and adriamycin through a mechanism unrelated to p21 and dependent on p53. DNA Repair 8: 390-399, 2009. 